\title{
Severe maxillofacial renal osteodystrophy in two patients with chronic kidney disease
}

\author{
Maria Luiza Diniz de Sousa Lopes ${ }^{1}$ - Assis Filipe Medeiros Albuquerque ${ }^{2}$. \\ Adriano Rocha Germano ${ }^{2}$ - Lélia Maria Guedes Queiroz ${ }^{1}$. \\ Márcia Cristina da Costa Miguel ${ }^{1} \cdot$ Éricka Janine Dantas da Silveira ${ }^{1}$
}

Received: 7 December 2014 / Accepted: 9 March 2015 / Published online: 19 March 2015

(C) Springer-Verlag Berlin Heidelberg 2015

\begin{abstract}
Renal osteodystrophy (ROD) is the bone pathology that occurs as an uncommon complication related to the several alterations in mineral metabolism present in patients with chronic kidney disease (CKD). This paper describes two cases of severe ROD affecting the maxilla and mandible and causing facial disfigurement of a young and a middle-aged female patient with CKD. Both patients had a history of secondary hyperparathyroidism, previously treated by surgery. The pathogenesis of the disease, as well as its clinical, imaging, and histopathological features, and management of the patient are discussed.
\end{abstract}

Keywords Renal osteodystrophy - Chronic kidney disease . Secondary hyperparathyroidism $\cdot$ Chronic kidney disease-mineral bone disorder

\section{Introduction}

Chronic kidney disease (CKD) is the progressive and irreversible loss of renal function, usually leading to end-stage renal disease, a potentially fatal condition, requiring chronic hemodialysis or kidney transplantation for survival $[1,2]$. One of the complications that can arise in patients with CKD is the clinical syndrome chronic kidney disease-mineral and bone

Maria Luiza Diniz de Sousa Lopes

marialu.diniz@gmail.com

1 Postgraduate Program in Oral Pathology, Dentistry Department, Federal University of Rio Grande do Norte, Avenida Salgado Filho, 1787-Lagoa Nova, Natal, RN CEP 59.056-000, Brazil

2 Oral and Maxillofacial Surgery and Traumatology Residency, Dentistry Department, Federal University of Rio Grande do Norte, Natal, RN, Brazil disorder (CKD-MBD), which involves changes in laboratory tests such as serum calcium, organic phosphate, parathyroid hormone (PTH), and concentrations of vitamin D derivatives; changes in bone turnover; and extraskeletal calcifications [3, 4]. Renal osteodystrophy (ROD) has recently been redefined to refer to the bone pathology from this syndrome [5].

Advancements in the knowledge about CKD and its complications, such as secondary hyperparathyroidism (HPT-II) and ROD, resulted in a decreased frequency of significant macroscopic bone alterations in kidney patients. Nonetheless, radiographic changes of the facial skeleton still eventually occur, occasionally involving the jaws, and may represent an early sign of ROD [6-8]. This manuscript reports the details of two unusually severe maxillofacial manifestations of ROD in patients with CKD, emphasizing the laboratorial, clinical, imaging, and histological features as well as patient management.

\section{Case reports}

\section{Patient 1}

A 23-year-old female was referred to the Oral and Maxillofacial Surgery Service of our department with a 4year history of an asymptomatic, expansive mass in the right side of the face causing airway compromise and facial disfigurement (Fig. 1a). On intraoral examination, there were macroscopic enlargement of the right maxilla and a less severe increase in volume of the right mandible (Fig. 1b). In addition, the patient presented gingivitis, carious teeth, and malocclusion. She had a history of idiopathic CKD in advanced stage and had been receiving hemodialysis three times a week for 10 years, while on a kidney transplant waiting list. During this period, she developed HPT-II reaching $1452 \mathrm{pg} / \mathrm{mL}$ as a 
Fig. 1 Extraoral view showing a severe swelling associated with facial asymmetry and deletion of the right nasolabial fold (a). Intraoral view showing the wellcircumscribed enlargement in the right maxilla causing displacement of adjacent teeth and a smaller swelling in the edentulous area of the mandible (b)
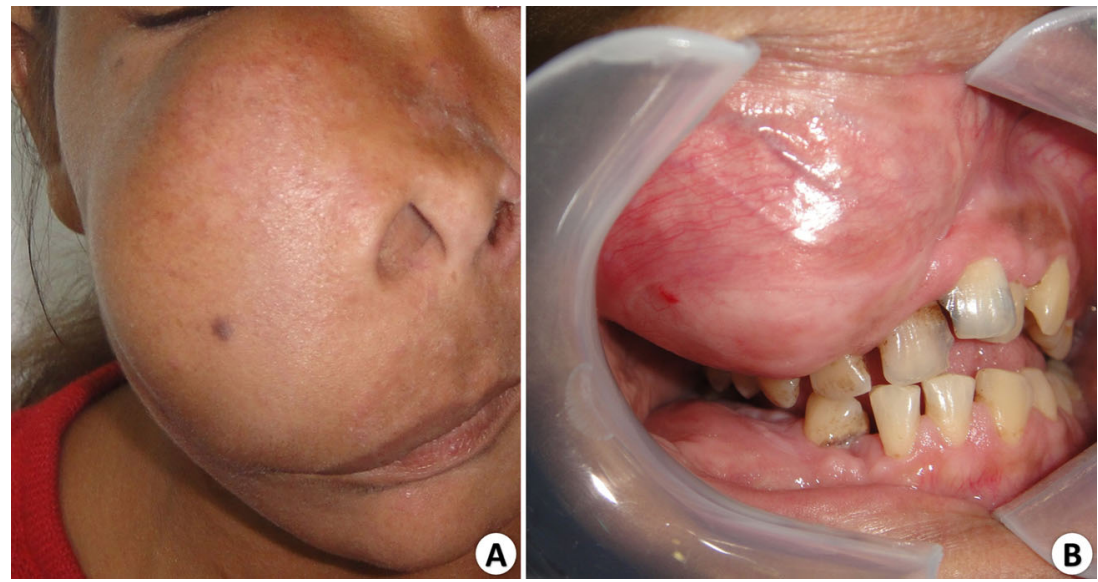

maximum level of PTH. The treatment was a total parathyroidectomy combined with partial autotransplantation in the forearm. Subsequently, she reported that the facial lesion size stabilized. Her current medication included folic acid, B vitamins, prednisone, Renagel, and calcium carbonate. The panoramic radiography demonstrated a prominent ill-defined radiopaque area in the maxilla affecting the maxillary sinus and a mixed radiolucency-radiopacity in the mandible. Both areas displayed a ground-glass appearance (Fig. 2a). The clinical diagnosis of a brown tumor was suggested. Incisional biopsy of the maxillary affected area was performed under local anesthesia, and microscopic examination showed interconnected and irregularly shaped trabeculae of woven bone interspersed with loose connective tissue with sparse fibroblasts and moderate vascularization (Fig. 2b). The histopathological diagnosis was suggestive of renal osteodystrophy. A computed tomography (CT) scan and blood tests were requested in order to plan the surgical removal of the lesion. The CT scan revealed marked osseous expansion on the right side of the face, with endophytic and exophytic growth, affecting the body of the zygomatic bone and the entire portion of the right maxilla (Fig. 3a). The involvement of the infraorbital floor and invasion of the nasal cavity were observed, which resulted in nasal and ophthalmic functional commitment, respectively. A lateral radiograph of the thoracic spine showed an intact bone structure (Fig. 3b), and there was no reported involvement of the limbs. Abdominal, cardiovascular, and pulmonary examinations were unremarkable. Laboratorial findings, 3 days before the surgical procedure, showed a red blood cell count of 5.7 cells $/ \mathrm{mm}^{3}$ (normal range 4-5.2 cells $/ \mathrm{mm}^{3}$ ), leukocyte count of $6.400 / \mu \mathrm{L}$ (normal $3.500-10.000 / \mu \mathrm{L}$ ), platelet count of $104.000 / \mathrm{mm}^{3}$ (normal $150.000-450.000 / \mathrm{mm}^{3}$ ), hematocrit of $45.2 \%$ (normal range for women 35-46\%), serum creatinine of $11.19 \mathrm{mg} / \mathrm{dL}$ (normal $0.53-1.2 \mathrm{mg} / \mathrm{dL}$ ), serum calcium of $7.01 \mathrm{mg} / \mathrm{dL}$ (normal $8.8-11 \mathrm{mg} / \mathrm{dL}$ ), serum phosphate of $4.72 \mathrm{mg} / \mathrm{dL}$ (normal range $2.5-4.8 \mathrm{mg} / \mathrm{dL}$ ), alkaline phosphatase level of 89 international units (IU)/L (normal range 27-100 IU/L), and serum PTH of $149 \mathrm{pg} / \mathrm{mL}$ (normal 10$65 \mathrm{pg} / \mathrm{mL}$ ). The patient was submitted to surgical removal of the lesion at the hospital under general anesthesia with orotracheal intubation. One day prior to the procedure, the patient underwent hemodialysis, and due to the CKD status and thrombocytopenia, a hemodynamic preparation was conducted preoperatively and in the immediate postoperative
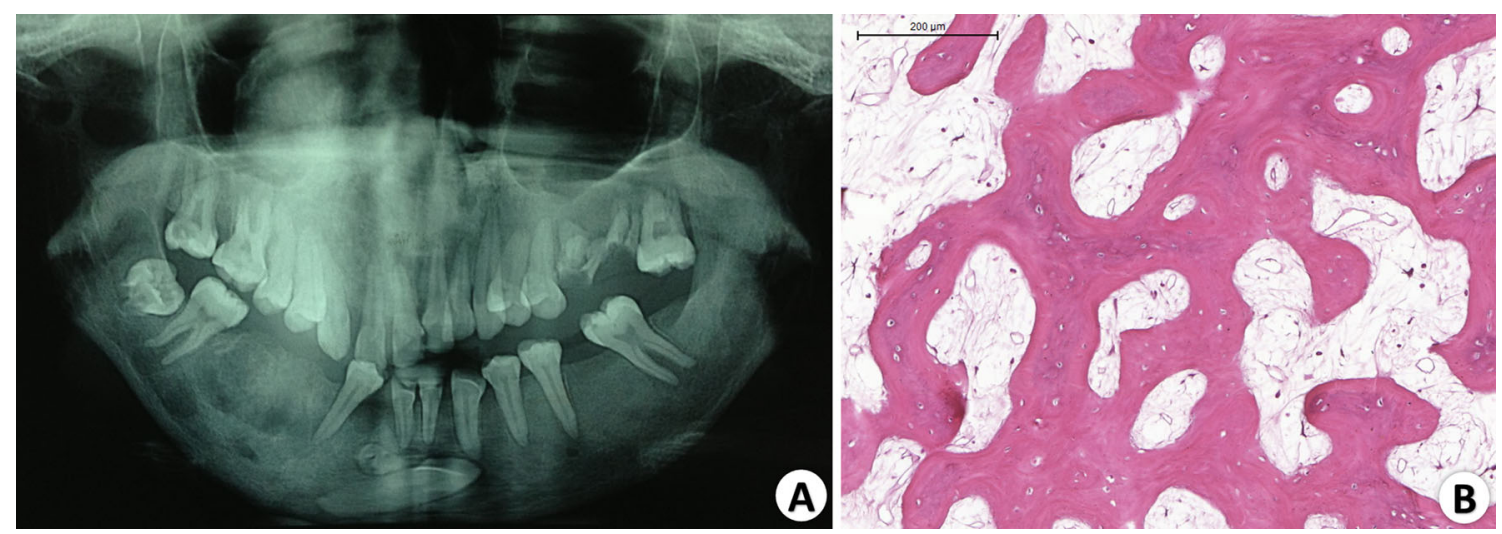

Fig. 2 Radiopaque image with imprecise limits in the anterior maxilla involving the maxillary sinus. Mandibular lesion revealing a mix of radiolucent and radiopaque appearance and well-defined limits (a).
Histological appearance revealing trabeculae of vital woven bone lying in a loose connective tissue background containing few cells and moderate vascularization $(\mathrm{H} \& \mathrm{E})(\mathbf{b})$ 
Fig. 3 Axial CT scan showing bone expansion consisting of irregularly arranged bone trabeculae with considerable variation in density (a). Lateral radiograph of the thoracic spine showing no bone alterations (b)
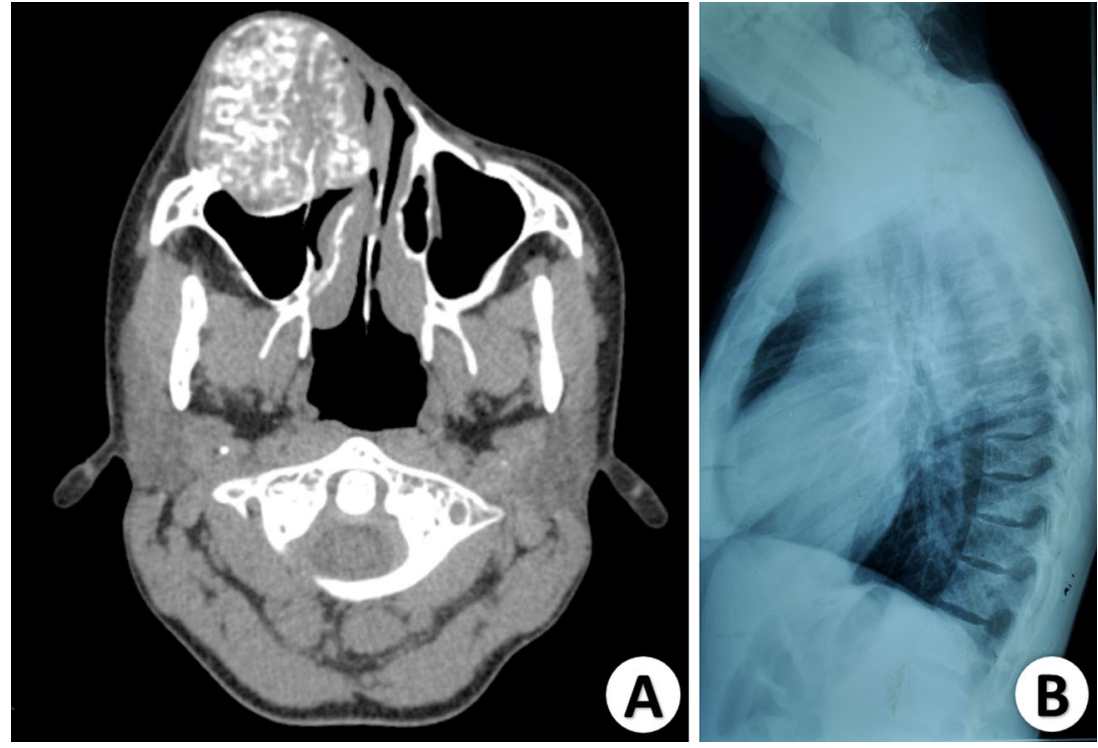

period. Hence, the infiltration of 2 bags of platelet-rich plasma and 10 units of cryoprecipitate was used as suggested by the hematology service of the hospital. During surgery, a WeberFerguson incision was made, followed by dissection in planes (maintaining the integrity of the oral mucosa and attached gingiva) to obtain a proper visualization of the lesion (Fig. 4a). Due to the extension of the lesion far to the medial, reaching the nasal cavity, orbital floor, hemi-maxilla, and zygomatic bone, only the osteoplasty and plasty of the surrounding soft tissue were performed, avoiding the mutilation of the patient and restoring the normal conformation of the face. The lesion invading the region of the nasal cavity was removed, in order to improve the breathing. After the procedure, the patient went to the intensive care unit and underwent hemodialysis 1 day after surgery. The fragments sent for pathological analysis revealed the same characteristics of the specimen previously obtained from incisional biopsy. The final pathologic diagnosis was consistent with ROD. One year after the surgery (Fig. 4b), the patient showed no evidence of any increase of volume in the maxilla and is preparing for the removal of the mandibular lesion, as well as for the kidney transplant.

\section{Patient 2}

A 44-year-old woman presented an extraoral appearance of significant swelling in the middle and lower third of the face (Fig. 5a); thus, the clinical description of "leontiasis ossea" was presented. On intraoral examination, a hard and asymptomatic swelling in the right maxilla was evident, as well as in the mentonian area of the mandible, both covered by normal mucosa (Fig. 5b). The maxillary lesion was expanding the buccal bone plate along with the hard palate. In the mandible, significant expansion in the buccal bone plate mandible involving the region between teeth 36 and 46 was observed, changing the normal teeth arrangement. The patient complained of difficulty breathing through the nose at night. The patient first noted the enlargement 6 years earlier which steadily increasing since then. Medical history showed hypertension and a 10-year history of CKD for which she has been
Fig. 4 Once the lesion was exposed during surgery, it revealed bleeding aspect (a). Postoperative appearance 1 year after surgery (b)
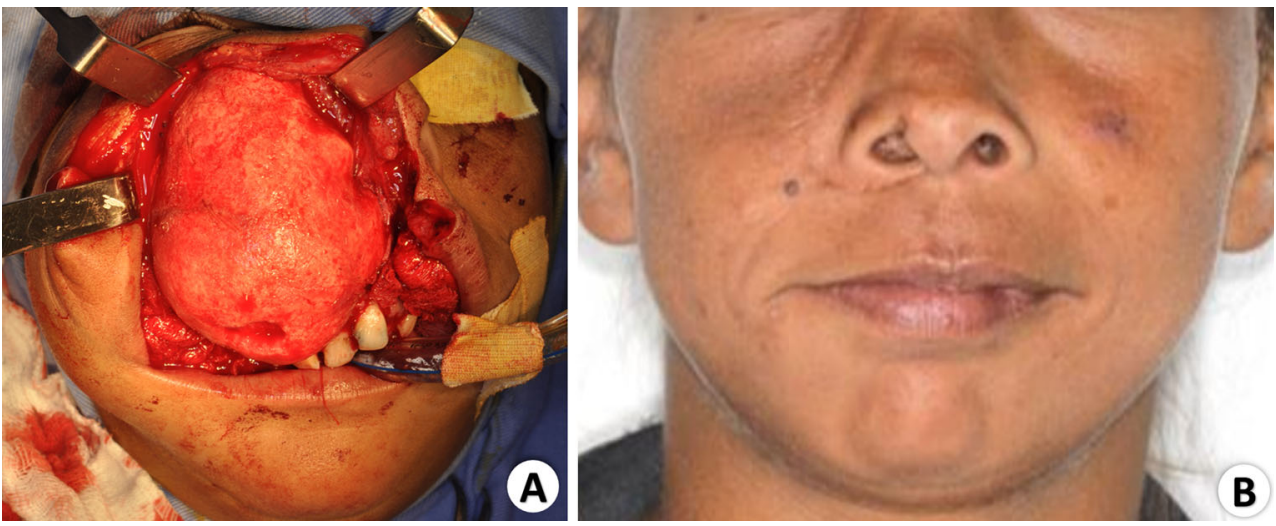

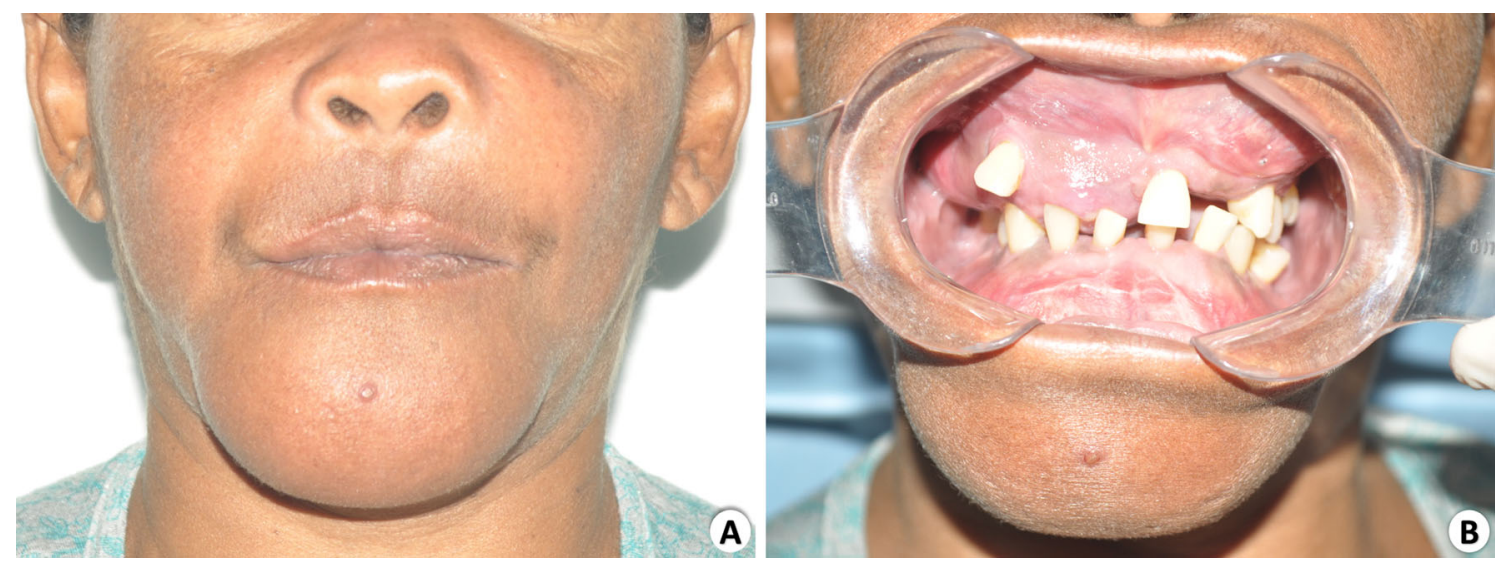

Fig. 5 Extraoral view (patient 2) showing a swelling in the middle and lower third of the face resembling a leonine face (a). Intraoral view of severe maxillary and mandibular enlargement causing teeth separation (b)

receiving hemodialysis three times a week. During this period, the patient developed HPT-II, achieving a maximum value of serum parathyroid hormone (PTH) of $1056 \mathrm{pg} / \mathrm{mL}$ (normal $10-65 \mathrm{pg} / \mathrm{mL})$. The patient underwent total parathyroidectomy associated with partial autotransplantation in the forearm 1 year before being referred to our department. Her medication included losartan potassium and clonidine hydrochloride. Panoramic radiography showed a marked expansion of the maxilla and mandible, with a ground-glass appearance and teeth hypercementosis (Fig. 6). There were no bone manifestations at other areas of the body. Laboratory examination showed current red blood cell count of 4.2 cells $/ \mathrm{mm}^{3}$ (normal range 4-5.2 cells $/ \mathrm{mm}^{3}$ ), leukocyte count of $6.200 / \mu \mathrm{L}$ (normal $3.500-10.000 / \mu \mathrm{L}$ ), platelet count of $325.000 / \mathrm{mm}^{3}$ (normal $150.000-450.000 / \mathrm{mm}^{3}$ ), hematocrit of $37.8 \%$ (normal range for women $35-46 \%$ ), serum creatinine of $11.17 \mathrm{mg} / \mathrm{dL}$ (normal $0.53-1.2 \mathrm{mg} / \mathrm{dL}$ ), serum calcium of $6.9 \mathrm{mg} / \mathrm{dL}$ (normal $8.8-11 \mathrm{mg} / \mathrm{dL}$ ), serum phosphate of $4.72 \mathrm{mg} / \mathrm{dL}$ (normal range $2.5-4.8 \mathrm{mg} / \mathrm{dL}$ ), alkaline phosphatase of $244 \mathrm{IU} / \mathrm{L}$ ) (normal range 27-100 IU/L), and serum PTH of $208 \mathrm{pg} / \mathrm{mL}$ (normal 10-65 pg/mL). The clinical diagnostic hypothesis was ROD and brown tumor. The patient underwent incisional biopsies of both maxillary and mandibular lesions under local anesthesia. Microscopic examination revealed vital and

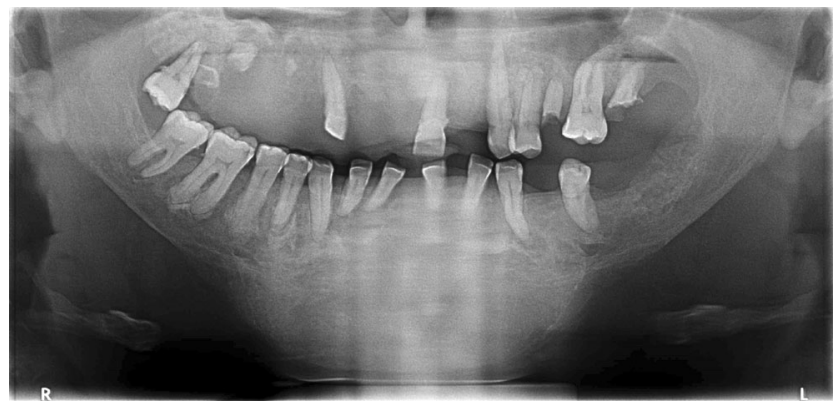

Fig. 6 Panoramic radiography (patient 2) displaying marked expansion of bone structures with ground-glass appearance and several teeth with hypercementosis irregular trabeculae of woven bone in different maturation stages, occasionally showing osteoblastic rimming as well as multinucleated osteoclast-type giant cells into a vascularized fibrous connective tissue (Fig. 7). The clinical, biochemical, imaging, and microscopic studies lead to a diagnosis of osteitis fibrosa consistent with ROD. The patient was submitted to osteoplasty of both lesions by an intraoral approach in the hospital under general anesthesia. Sections of the collected specimen confirmed the histopathological diagnosis osteitis fibrosa consistent with ROD. Ten months after the surgery, the patient shows no noticeable bone changes on her facial contour and is under nephrologic care (Fig. 8).

\section{Discussion}

Patients with CKD commonly have alterations in several mineral metabolisms including hyperphosphatemia, hypocalcemia, and 1,25-dihydroxyvitamin $\mathrm{D}_{3}$ or calcitrol (active metabolite of vitamin D), which eventually lead to a compensatory overproduction of PTH by parathyroid glands in a condition known as HPT-II [9-11]. As renal function declines, 1,25dihydroxyvitamin $\mathrm{D}_{3}$ synthesis becomes impaired, which

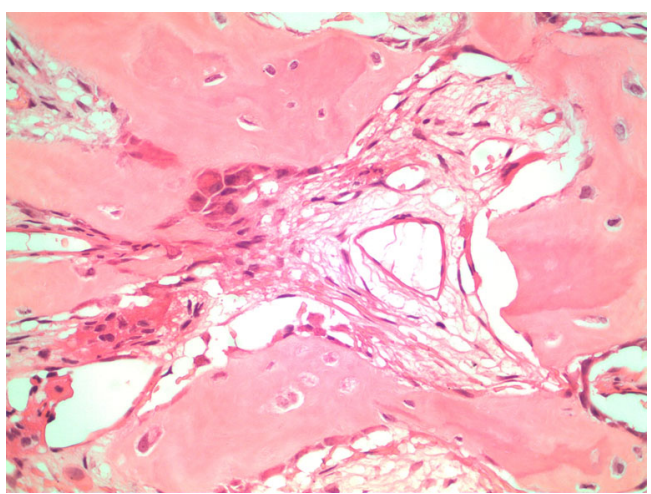

Fig. 7 Multinucleated giant cells throughout the stroma and plump osteoblasts adjacent to the osteoid matrix (patient 2; H\&E) 


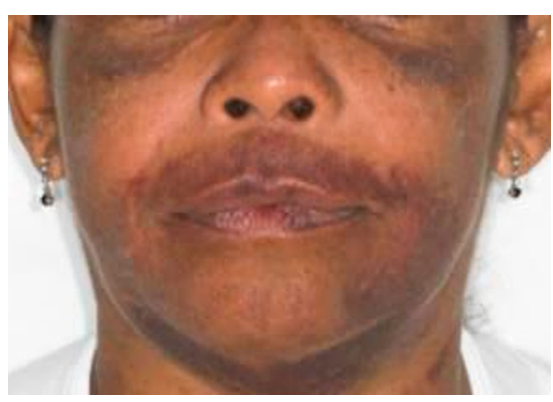

Fig. 8 Facial appearance 10 months after operation (patient 2)

leads to deficiency in renal calcium absorption, resulting in hypocalcemia. The calcium sensing receptor (CaSR), which is highly expressed in the parathyroid glands, senses the low serum calcium concentrations, leading to a compensatory increase in PTH (HPT-II) in the attempt to maintain the homeostasis of calcium [10]. In the meantime, the reduction of glomerular filtration also leads to impaired phosphate excretion, elevating the serum concentration of phosphate. Hyperphosphatemia can act by directly stimulating PTH synthesis and parathyroid hyperplasia and indirectly promoting HPT-II by decreasing the serum calcium level $[1,2,10]$. Excess of PTH acts in the transformation of the inactive form of vitamin $\mathrm{D}$ to 1,25 -dihydroxyvitamin $\mathrm{D}_{3}$ in the kidney and influences the development and activity of osteoclasts, osteoblasts, and osteocytes, thereby affecting bone turnover [1, 4].

Due to the mineral alterations, patients with ROD should run basic blood tests, including electrolytes, full blood cell count, PTH, and alkaline phosphatase levels [2]. The laboratory findings of both patients revealed a serum PTH level slightly elevated, but when it is compared to the levels before the removal of the parathyroid glands, the considerable improvement in the HPT-II status became evidently unmistakable. Especially in patient 1, this fact was supported by the demonstrated normal levels of calcium, phosphorus, and alkaline phosphatase, the latter being a biochemical marker and, together with the PTH, commonly used in the diagnosis of different forms of bone disease associated with CKD [12].

Usually, the alterations in bone metabolism are diagnosed by laboratory tests before the development of macroscopic skeletal changes $[7,10]$. The majority of macroscopic manifestations of ROD present as diffuse jaw enlargement [13]. However, recently, the pattern of localized expansion was reported to be more common than originally recognized [5]. Patient 1 exhibited localized enlargements of the maxilla and mandible, causing facial disfigurement, while the bone alterations of patient 2 looked more diffuse. In addition, the alterations were exclusively in the jaw bones, any manifestation in long bones and aorta being discarded.

The extreme clinical manifestation of ROD is denominated leontiasis ossea represented by hyperostotic changes in the facial bones and may cause an expansion of the malar processes, a reduced nasomaxillary angle, widening of the nares, flattening of the nasal bridge, and an increase of interdental space [9]. This ultimate condition results in lion-like facial features observed in patient 2 , which clinically and histologically resembles the big head disease observed in animals with hyperparathyroidism secondary to kidney problems or to diets rich in phosphorus and calcium deficiency [2].

Such facial disfigurement as in leontiasis ossea is an uncommon feature of ROD and is not specific because it can be present in other lesions such as fibrous dysplasia, or Paget's disease of bone [9]. Craniofacial fibrous dysplasia is asymmetric and can cause cranial neuropathies, headaches, and occlusal discrepancies [2]. In contrast to ROD, fibrous dysplasia typically occurs in childhood and accompanies a biochemical profile within normal limits $[5,13]$. Concerning Paget's disease, the rates of osteoclastic and osteoblastic activities are elevated, resulting in skeletal remodeling and weakened deformed bones. However, when it affects jawbones, it is typically bilateral [14]. Moreover, even showing increased levels of alkaline phosphatase, the serum level of calcium in Paget's disease is normal [2].

Some radiographic findings of ROD of the jaws also occur in fibrous dysplasia, or Paget's disease of bone, such as ground-glass appearance, observed in both current patients; hypercementosis noticed on patient 2; and bone resorption with loss of lamina dura [13]. Some of the radiographic alterations of ROD include demineralization of bone, delayed tooth eruption, mixed osteolysis and sclerosis (salt-and-pepper) trabecular appearance, absence of lamina dura, thickening of the diploic space of the calvarium, dental spacing and mobility, jaw fracture, and poor definition of anatomical landmarks [9]. The CT scan can show changes in the trabecular pattern and bone expansion and, along with magnetic resonance imaging, is helpful for diagnosis and treatment planning [15].

Microscopic and pathologic features of ROD are dynamic in their progression and can be subdivided into low- and highturnover bone diseases or with a combined pattern of the two categories [16]. Osteitis fibrosa represents increased bone turnover as it is associated with hyperparathyroidism, while decreased turnover mainly characterizes osteomalacia and adynamic (aplastic) bone disease [7, 13]. Osteitis fibrosa used to be the most common type of ROD; however, in the last decades, adynamic bone disease has become the most common form [17]. Microscopic findings of osteitis fibrosa reveal prominent peritrabecular fibrosis associated with an increased number and activity of osteoclasts associated with increased numbers of osteoblasts and osteoid $[2,13,18]$. Those features were present in patient 2 , justifying the diagnosis of osteitis fibrosa. On the other hand, osteomalacia is characterized by defective mineralization and increased osteoid production mainly caused by the decrease of 1,25-dihydroxyvitamin $\mathrm{D}_{3}$ concentrations, usually causing osseous pain and musculoskeletal disability and making the patient at risk of osteopenia, 
bone deformity, and fracture $[1,4,5]$. Adynamic bone disease is most frequent in CKD patients without HPT-II and is usually related to increased risk of hypercalcemia, cardiovascular calcification, and mortality. No osteoid accumulation is present, and there are only few or absent osteoblasts and little or no fibrosis in either the bone marrow or peritrabecular area [17]. The absence of osteoid deposition or osteoclastic activity in patient 1 specimens reflects the lack of active bone metabolism, or even normal bone metabolism, probably because the patient's bone disease was under control.

Differential diagnosis of ROD, besides fibrous dysplasia and Paget's disease of the bone, should include brown tumors of HPT-II, which generally can be discarded by radiographic appearance or/and histopathological examination. Brown tumors are more likely to occur in primary hyperparathyroidism, showing a radiographic appearance of a well-circumscribed radiolucent lesion and typically revealing multinucleated giant cells and prominent hemosiderin deposition under light microscopy $[5,6,8]$. As observed in patient 2 , the presence of multinucleated giant cells can also occur in ROD, but according to Lerman et al. [5], it is less prominent than brown tumors. The Sagliker syndrome, recently described by Sagliker et al. [19], also must be considered in the differential diagnosis of ROD, since it is seen in $0.5 \%$ of patients with CKD [20]. However, in addition to HPT-II and CKD, the diagnostic criteria for this syndrome include short stature, extremely severe maxillary and mandibular changes (uglifying the face), soft and histopathologically verified benign epithelial hyperplasia, severe teeth/dental abnormalities, fingertip changes, knee and scapula deformities, abnormalities in audition, and severe neurological and psychological disturbances [20, 21].

Management of ROD is based on control of factors responsible for the development of altered bone metabolism [22]. ROD associated with HPT-II can be improved with the correction of calcium and vitamin $\mathrm{D}$ levels and a decrease in phosphate levels. This can be accomplished by conservative treatment, such as the use of phosphate-binding agents, treatment with calcitriol and other active vitamin D sterols, and following a phosphate-restricted diet [1, 2, 9]. Despite medical therapy, in several patients, the altered PTH levels persist, requiring surgical intervention with total parathyroidectomy with or without autotransplantation or subtotal parathyroidectomy. The ultimate treatment for persistent HPT-II in the two current patients was parathyroidectomy with autotransplantation, which involves the transplantation of a small amount of parathyroid tissue into the forearm, aiming to maintain normal parathyroid function. If recurrent hyperparathyroidism occurs, this technique allows a simple access, with locoregional anesthesia, for removal of the fragments from the forearm [23].

Even with the adjustment of the PTH levels, the total regression of bone alterations is unusual; therefore, most cases require surgical recontouring. There are few reports of ROD manifestations in jaws resolved after the correction of the HPT-II condition, without surgical intervention [24, 25]. The jaw enlargement in the present patients ceased after the treatment of HPT-II, but surgery was necessary mainly for improvement of breathing and for esthetics consideration.

This manuscript emphasizes the importance of a detailed anamnesis and clinical and microscopic investigation for the diagnosis and appropriate treatment of ROD affecting the maxillofacial region. Even though the altered bone metabolism usually is biochemically detected before patients have any significant symptomatology, the cases reported here highlight that in unusual circumstances it may develop into severe bone alterations.

Conflict of interest The authors declare no conflict of interest.

\section{References}

1. Antonelli JR, Hottel TL (2003) Oral manifestations of renal osteodystrophy: case report and review of the literature. Spec Care Dentist 23(1):28-34

2. Collum J, Jones RHB, Liynham A, Hirst J (2013) Leontiasis ossea: a presentation of hyperparathyroidism in an indigenous Australian man secondary to chronic renal failure. J Oral Maxillofac Surg 71(1):56-61. doi:10.1016/j.joms.2012.04.008

3. Chauhan V, Kelepouris E, Chauhan N, Vaid M (2012) Current concepts and management strategies in chronic kidney diseasemineral and bone disorder. South Med J 105(9):479-485. doi:10. 1097/SMJ.0b013e318261f7fe

4. Lim CY, Ong KO (2013) Various musculoskeletal manifestations of chronic renal insufficiency. Clin Radiol 68(7):e397-e411. doi:10. 1016/j.crad.2013.01.025

5. Lerman MA, Do C, Gunaratnam L, Kulkarni C, Tucker K, Woo SB (2012) Localized mandibular enlargement in end-stage renal disease: two case reports and a review of the literature. Oral Surg Oral Med Oral Pathol Oral Radiol 113(3):384-390. doi:10.1016/j. tripleo.2011.04.039

6. Damm DD, Neville BW, McKenna S, Jones AC, Freedman PD, Anderson WR et al (1997) Macrognathia of renal osteodystrophy in dialysis patients. Oral Surg Oral Med Oral Pathol Oral Radiol Endod 83(4):489-495

7. Chang JI, Som PM, Lawson W (2007) Unique imaging findings in the facial bones of renal osteodystrophy. Am J Neuroradiol 28(4): 608-609

8. Karsburg RM, Campos KR, Peres MPSM, Bologna SB, Lourenço SV, Franco JB (2012) Facial lesions caused by renal osteodystrophy in a patient with chronic renal insufficiency: a case report. Rev Odontol Cienc 27(2):161-165

9. Fatahzadeh M (2011) The spectrum of orofacial manifestations in renal osteodystrophy: diagnostic challenges of an uncommon presentation. Quintessence Int 42(7):e78-e88

10. Heymann EP, Jenkins M, Goldsmith D (2011) Clinical features and manifestations of CKD-MBD. Clin Rev Bone Miner Metab 10(3): 142-148

11. Way FM, Lessard M, Lafage-Proust MH (2012) Pathophysiology of chronic kidney disease-mineral and bone disorder. Joint Bone Spine 79(6):544-549 
12. Martin KJ, Olgaard K, Coburn JW, Coen GM, Fukagawa M, Langman C et al (2004) Bone Turnover Work Group: diagnosis, assessment, and treatment of bone turnover abnormalities in renal osteodystrophy. Am J Kidney Dis 43(3):558-565

13. Kalyvas D, Tosios KI, Leventis MD, Tsiklakis K, Angelopoulos AP (2004) Localized jaw enlargement in renal osteodystrophy: report of a case and review of the literature. Oral Surg Oral Med Oral Pathol Oral Radiol Endod 97(1):68-74

14. Brooks JK, Rivera-Ramirez LE, Errington LW, Scheper MA (2011) Synchronous Paget disease of bone and hyperparathyroidism: report of a case with extensive craniofacial involvement. Oral Surg Oral Med Oral Pathol Oral Radiol Endod 111(4):e19-e24. doi:10. 1016/j.tripleo.2010.11.004

15. Abdel Razek AA (2014) Computed tomography and magnetic resonance imaging of maxillofacial lesions in renal osteodystrophy. $\mathrm{J}$ Craniofac Surg 25(4):1354-1357. doi:10.1097/SCS. 0000000000000819

16. Thomas R, Kanso A, Sedor JR (2008) Chronic kidney disease and its complications. Prim Care 35(2):329-344

17. Morrow B, Qunibi W (2012) Specific bone and mineral disorders in patients with chronic kidney disease. Clin Rev Bone Miner Metab 10(3):184-208

18. Adachi PLG, Santos PSS, Magalhães MHCG, Martins MT (2007) Renal osteodystrophy manifesting as localized enlargement of the jaw. Nephrol Dial Transplant 22:2398-2399. doi:10.1093/ndt/ gfm 232
19. Sagliker Y, Balal M, Ozkaynak PS, Paydas S, Sagliker C, Sagliker HS et al (2004) Sagliker syndrome: uglifying human face appearance in late and severe secondary hyperparathyroidism in chronic renal failure. Semin Nephrol 24(5):449-455

20. Yavascan O, Kose E, Alparslan C, Kose SS, Bal A, Kanik A et al (2013) Severe renal osteodystrophy in a pediatric patient with endstage renal disease: Sagliker syndrome? J Ren Nutr 23(4):326-330. doi:10.1053/j.jrn.2012.07.002

21. Giray S, Sagliker Y, Yildiz I, Halvaci I, Paylar N, Ocal F et al (2006) Neurologic manifestations in Sagliker syndrome: uglifying human face appearance in severe and late secondary hyperparathyroidism in chronic renal failure patients. J Ren Nutr 16(3):233-236

22. Malluche HH, Mawad HW, Monier-Faugere MC (2011) Renal osteodystrophy in the first decade of the new millennium: analysis of 630 bone biopsies in black and white patients. J Bone Miner Res 26(6):1368-1376. doi:10.1002/jbmr.309

23. Conti-Freitas LC, Foss-Freitas MC, Lucca LJ, da Costa JA, Mamede RC, Foss MC (2009) Dynamics of parathyroid hormone secretion after total parathyroidectomy and autotransplantation. World J Surg 33(7):1403-1407. doi:10.1007/s00268-009-0057-8

24. Adornato MC, Mayne RW (2000) Macrognathia of renal osteodystrophy in a dialysis patient. Report of a case. N Y State Dent J 66(10):30-34

25. Hata T, Irei I, Tanaka K, Nagatsuka H, Hosoda M (2006) Macrognathia secondary to dialysis-related renal osteodystrophy treated successfully by parathyroidectomy. Int J Oral Maxillofac Surg 35(4):378-382 